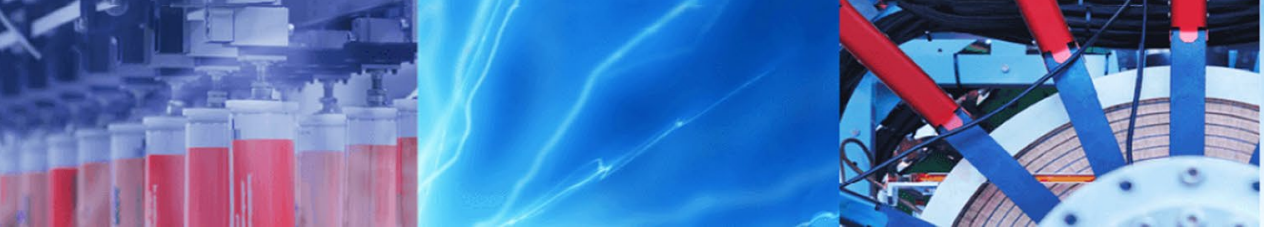

Research Article

\title{
Atherosclerosis research: the impact of physiological parameters on vascular wall stress
}

\author{
Sen Lu' ${ }^{1} \cdot$ Shusheng Zhang ${ }^{1}$ \\ (c) Springer Nature Switzerland AG 2019
}

\begin{abstract}
In the clinic, atherosclerosis has been associated with abnormal physiological conditions, such as high cholesterol level, high blood pressure and diabetes, but the mechanisms by which they are linked have not yet been determined. In recent years, hemodynamic factors have been found to have a great influence on atherosclerosis. In particular, wall shear stress (WSS), an important factor in atherosclerosis from the development of early lesions to the rupture of later plaques, is now one of the most important risk indicators of atherosclerosis. Here, we used a numerical investigation to determine the impact of four physiological parameters on vascular wall stress (including WSS and wall pressure) in the right coronary arteries, in order to assess their influence on atherosclerosis risk. Each parameter was investigated both separately and in integrated models. The results indicate that the blood flow rate has the most significant influence on WSS and plays a decisive role in the variation of WSS under normal physiological conditions. Blood pressure had a minor influence on WSS under conditions of a flexible vessel wall, but this effect was lost in the absence of flexibility. Blood viscosity and vascular elasticity could also affect WSS directly, but in vivo their influences were negligible.
\end{abstract}

Keywords Hydrodynamics · Wall shear stress · Blood pressure · Blood flow rate · Vascular elasticity

\section{Introduction}

Atherosclerosis is an inflammatory disease occurring principally in large-and medium-sized elastic and muscular arteries. It can lead to ischemia of the heart, brain or extremities, resulting in infarction [1]. Nowadays, it has become the leading cause of morbidity and mortality in the world [2]. The process of atherogenesis involves multiple systems and can last for decades; the earliest lesions are even found in infants and young children [3]. Finding the cause of atherosclerosis has become one of the toughest missions in modern medical research.

One of the important research questions is how atherosclerosis is linked with physiological conditions, such as blood pressure and viscosity. In previous investigations, it has been found that hypertension is associated with not only the formation of atherosclerosis $[4,5]$ but also the development of atherosclerotic plaques [6-8].
A three-year follow-up study [9] showed an additional $0.005-0.012 \mathrm{~mm} / \mathrm{y}$ progression of intima-media wall thickness (IMT) for every $\mathrm{mmHg}$ increase in blood pressure. There are also several lines of evidence suggesting that elevated blood viscosity can increase the risk of atherosclerosis [10-12]. All of these studies were conducted through clinical investigations; their results show a relationship between an abnormal physiological condition and atherosclerosis, but do not explain the mechanism by which this occurs.

Over recent decades, hemodynamic factors, particularly WSS [13-16], have been proven to play an important role in atherosclerosis. The changes to hemodynamics caused by physiological parameters could be the key to explaining the connection between abnormal physiological conditions and atherosclerosis risk. In order to explore this theory, we examined the impact of four common physiological parameters on VWS. These included blood pressure, blood flow rate,

Sen Lu, Is0628@mail.sdu.edu.cn | 'Institute of Thermal Science of Technology, Shandong University, Jinan, China.

SN Applied Sciences (2019) 1:692 | https://doi.org/10.1007/s42452-019-0737-4 
vascular elasticity and blood viscosity. They were investigated separately under steady flow and together under normal physiological conditions. The findings can provide important theoretical guidance and beneficial biophysical insights into atherosclerosis prevention and treatment.

\section{Materials and methods}

\subsection{Model geometry}

In blood vessels, the distribution of WSS is greatly affected by the vessel's geometry. To obtain accurate results, an accurate vessel model is required. Recently, medical image-based computational models have been used in many hemodynamics studies [17-19] due to their detailed nature. In this work, we used this technology to study the right coronary artery (RCA). The model, obtained from a 45-year-old healthy male, is shown in Fig. 1. In order to ensure the accuracy of the calculations, only the main branch (red line) was used. The internal diameter of the inlet is $3.7 \mathrm{~mm}$ and the outlet is $2.9 \mathrm{~mm}$. The wall thickness varies, measuring $20 \%$ of the internal diameter [20].

\subsection{Computational method}

To model the fluid-structure interactions (FSI), ANSYS-CFX software was used. High Resolution and Second Order Backward Euler were employed for advection scheme and transient scheme, respectively. The Navier-Stokes equations, coupled with elastodynamics equations, were applied simultaneously to describe the fluid domain, as well as the fluid-solid interactions [21]. The changes in the shape of the numerical domain, due to fluid-solid interactions, made remapping and re-meshing necessary during the numerical process, and large deflection theory was taken into consideration. The convergence criterion for all equations is $10^{-6}$, and they are solved using double precision. The mass and momentum conservation equations for an incompressible fluid can be written as: $\nabla \cdot \vec{v}=0$

$\rho\left(\frac{\partial \vec{v}}{\partial t}+\vec{v} \cdot \nabla \vec{v}\right)=-\nabla p+\nabla \cdot \vec{\tau}$

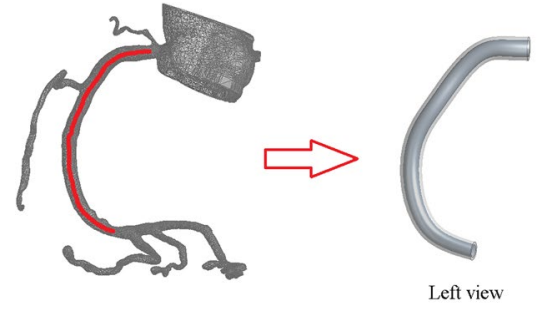

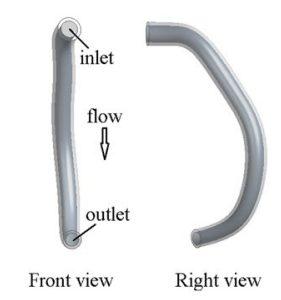

where $\rho$ is the density, $\vec{v}$ the velocity field, $p$ the pressure and $\vec{\tau}$ is the stress tensor, with a relation of $\vec{\tau}=\mu_{\text {app }} \cdot \overrightarrow{\dot{\gamma}}$. Here, $\mu_{\text {app }}$ is the apparent viscosity, depending on wall shear strain rate.

The equation describing the solid domain is:

$\rho_{s} \overrightarrow{a_{s}}=\nabla \overrightarrow{\sigma_{s}}+\overrightarrow{f_{s}}$

where $\rho_{s}$ is the density of the solid domain, $\overrightarrow{a_{s}}$ is the local acceleration of the solid domain, $\overrightarrow{\sigma_{s}}$ is the Cauchy stress tensor, and $\vec{f}_{s}$ is the body force vector.

The Carreau-Yasuda model $[22,23]$ was used to model the shear-thinning properties of blood.

$\mu=\mu_{\infty}+\left(\mu_{0}-\mu_{\infty}\right)\left[1+(\lambda \dot{\gamma})^{2}\right]^{\frac{n-1}{2}}$

where $\mu_{0}=0.056$ Pa s, $\mu_{\infty}=0.0035$ Pa s, $\lambda=3.313 \mathrm{~s}$, $n=0.3568[24,25]$.

\subsection{Boundary conditions}

The density of blood was $1060 \mathrm{~kg} / \mathrm{m}^{3}$, and the vessel wall was considered a linear elastic, isotropic and homogeneous material with a density of $1150 \mathrm{~kg} / \mathrm{m}^{3}$.

The default values for the four parameters, blood pressure, blood flow rate, vascular elasticity and blood viscosity, under steady flow, are shown in Table 1. To eliminate the influence caused by wall deformation when other parameters were studied, the default value for vascular elasticity was set to be $1000 \mathrm{MPa}$ - the vessel wall was assumed to be rigid. All the parameter values under steady flow were within normal physiological ranges.

The pulsatile blood flow waves and aortic pressure in the RCA are shown in Fig. 2. Blood pressure was used as the outlet boundary, while the blood flow rate was the inlet. Under normal physiological conditions, the heart rate was assumed to be $75 \mathrm{bpm}$.

\section{Results}

\subsection{Blood pressure}

As people age, blood vessels lose flexibility and become stiffer; one of the consequences of which is an increase in blood pressure. As atherosclerosis is associated with age, it is therefore possible that blood pressure is a contributing

Table 1 The default parameters under steady flow

\begin{tabular}{llll}
\hline $\begin{array}{l}\text { Blood pressure } \\
(\mathrm{KPa})\end{array}$ & $\begin{array}{l}\text { Blood flow rate } \\
(\mathrm{ml} / \mathrm{s})\end{array}$ & $\begin{array}{l}\text { Vascular elas- } \\
\text { ticity }(\mathrm{MPa})\end{array}$ & $\begin{array}{l}\text { Blood viscosity } \\
(\mathrm{Pa} \mathrm{s})\end{array}$ \\
\hline 16 & 1 & 1000 & 0.0035 \\
\hline
\end{tabular}

Fig. 1 The geometry of right coronary arteries 


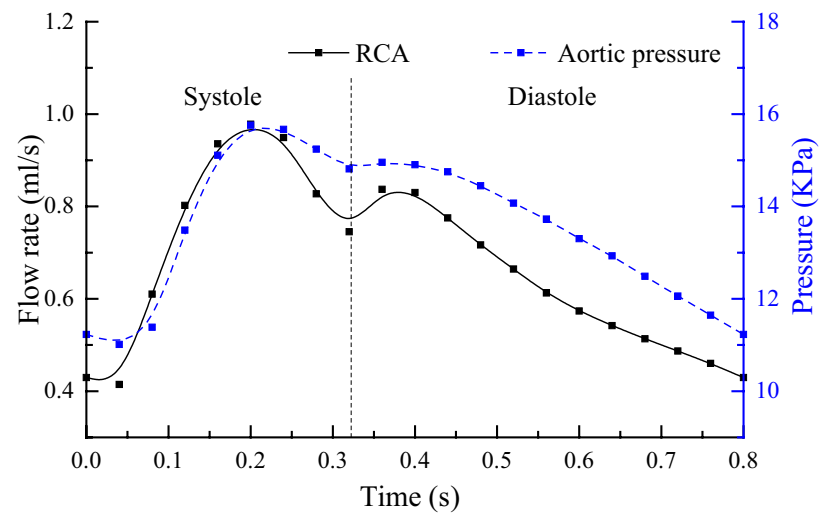

Fig. 2 Flow waveform used for boundary condition based on waveforms obtained from phase-contrast MRI velocity measurements in vivo [26]

factor. Keeping the other three parameters constant as in Table 1, the WSS distribution under different blood pressures was calculated and is shown in Fig. 3a. It can be seen that there were mainly two low-WSS regions in the system, which is in agreement with former research [27]. Region 1 was in the area where the direction of flow changed radically, and region 2 was on the inner wall of the curved segment. In particular, region 2 is a common location for atherosclerosis in patients. From the picture, it appears that the variation of blood pressure had no influence on WSS. The limit values of WSS in the system also verified this conclusion, as seen in Fig. 3b. The limit values for wall pressure are shown in Fig. 3c. The pressure difference between the upper limit (inlet) and lower limit (outlet) remain constant, about $0.15 \mathrm{kPa}$.

Although blood pressure had no influence on WSS, under normal conditions blood vessel walls are flexible, not rigid. Importantly, variations in blood pressure can affect the deformation of the vessel wall. Keeping vascular elasticity at $1 \mathrm{MPa}$, the WSS under different blood pressures was calculated and can be seen in Fig. 4a. In contrast to the rigid-wall model, the low-WSS region expanded notably when blood pressure increased. The limit values in Fig. $4 \mathrm{~b}$ demonstrate that WSS decreases as blood pressure increases. However, the effect was limited, about $0.036 \mathrm{~Pa}$ for every $\mathrm{kPa}$ increase. (a)

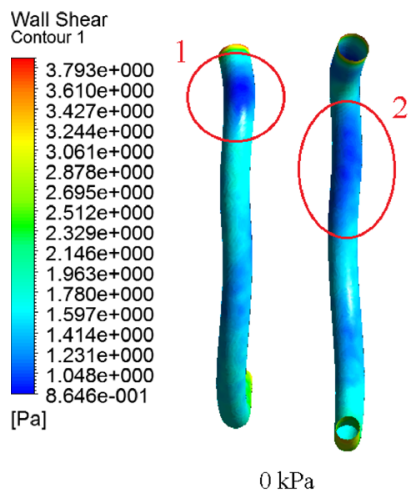

(b)

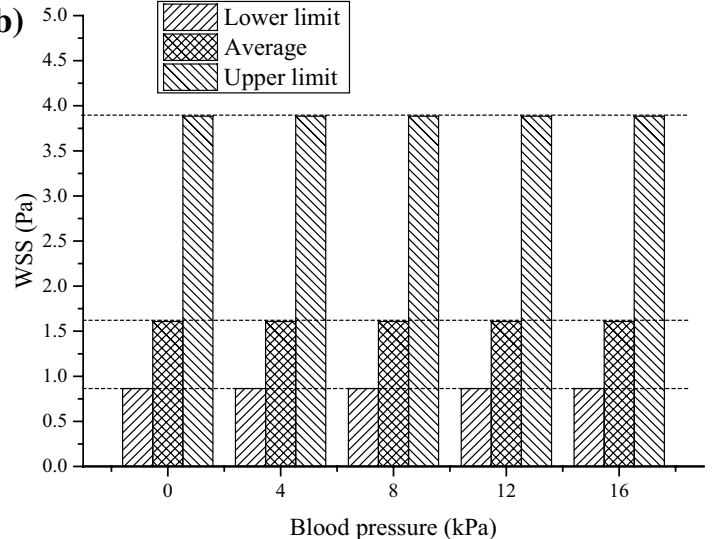

(1)

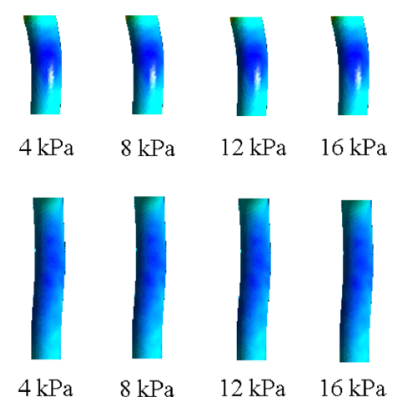

(c)

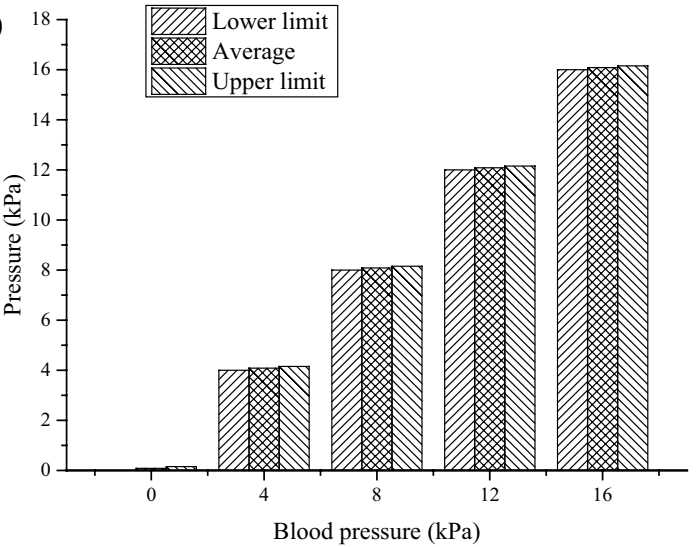

Fig. 3 VWS under different blood pressures in the rigid-wall model. a Distribution of WSS, $\mathbf{b}$ limit values for WSS, c limit values for wall pressure 


\subsection{Blood flow rate}

Figure 5 shows the VWS under different blood flow rates. As the flow rate increased, the WSS of the whole system increased rapidly. The lower limit of WSS increased by $1 \mathrm{~Pa}$ for every $\mathrm{ml}$ increase in blood flow rate. In Fig. 5c, it can be seen that a high blood flow rate can also increase wall pressure, which according to Fig. $4 \mathrm{~b}$ will indirectly decrease WSS in a normal deformable blood vessel. However, the effect of blood pressure on WSS was very limited, thus WSS is in proportion to blood flow rate.

\subsection{Vascular elasticity}

From the study of blood pressure, it can be seen that vascular elasticity could impact WSS. Besides aging, lifestyle factors can also affect the flexibility of vessel walls, such as diet, drinking and smoking. The loss of elasticity might contribute to the growing incidence of atherosclerosis among young people.

The distribution of WSS under different vascular elasticity is shown in Fig. 6a. As elasticity decreased, the areas of low-WSS region shrank. From Fig. 6b, it can be seen that as elasticity declined, the upper limit of WSS decreased and then increased, while the lower limit rose steadily and eventually leveled off at $0.86 \mathrm{~Pa}$. Both limits exhibited only minor changes, less than $0.5 \mathrm{~Pa}$, which could explain the limited influence of blood pressure under conditions of a flexible wall. Wall pressure also increased as vascular elasticity declined, which will further lessen its impact on WSS in normal arteries.

\subsection{Blood viscosity}

Figure 7 shows the influence of blood viscosity on VWS. Unlike the other three parameters, the change of blood viscosity affected the distribution of WSS. As viscosity increased, region 1 remained while region 2 perished. Similar to the effect of blood flow rate, increases in viscosity caused rapid increases in the limit values for WSS and wall pressure (Fig. 7b, c). (a)

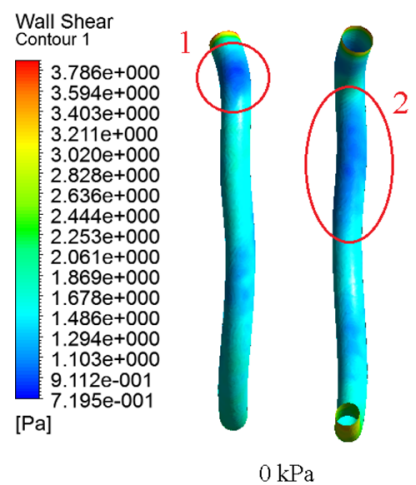

(b)

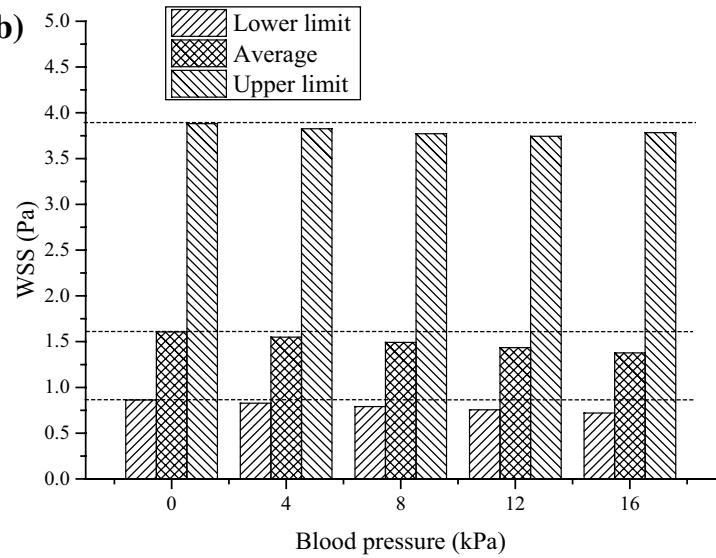

(1)
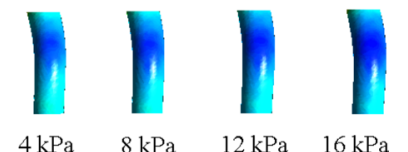

(2)
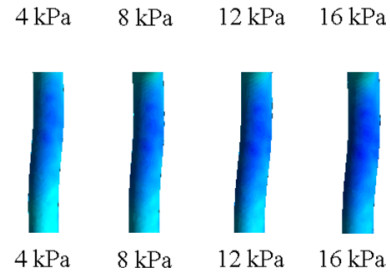

(c)

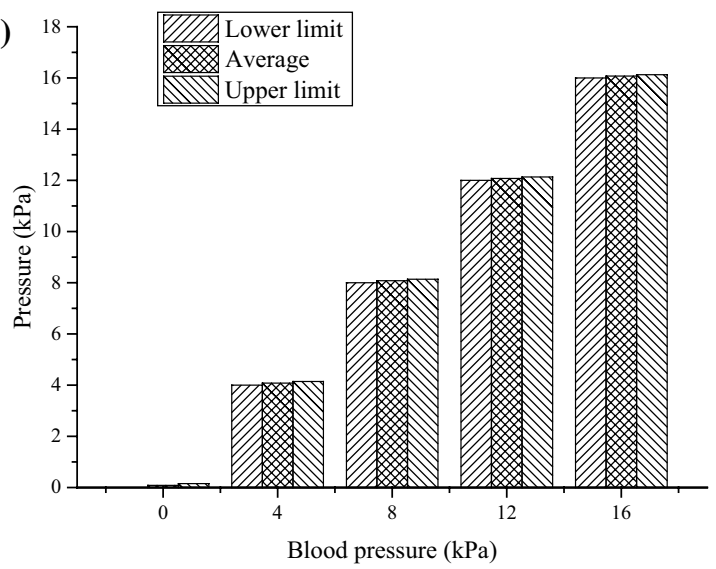

Fig. 4 VWS under different blood pressures in the deformable-wall model. a Distribution of WSS, $\mathbf{b}$ limit values for WSS, $\mathbf{c}$ limit values for wall pressure 
(a)

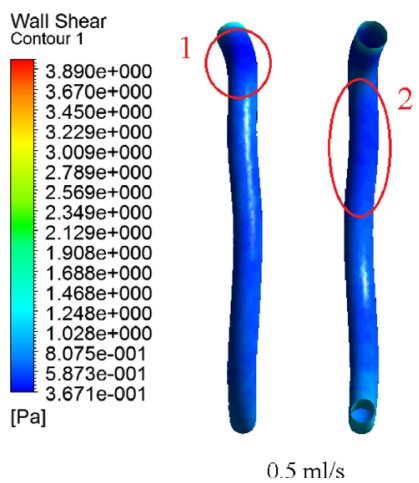

(1)

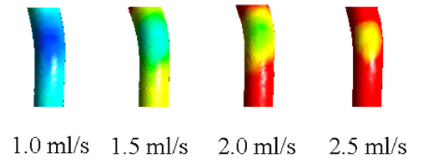

(2)

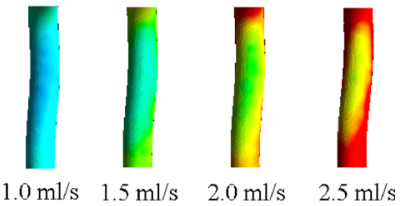

(b)

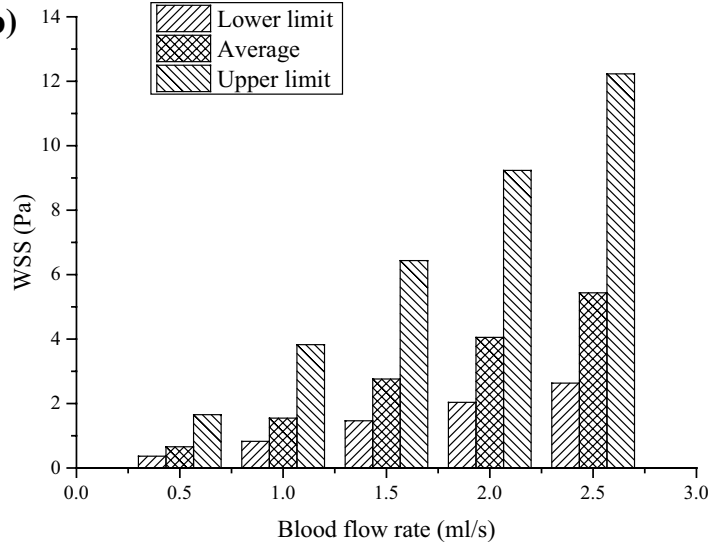

(c)

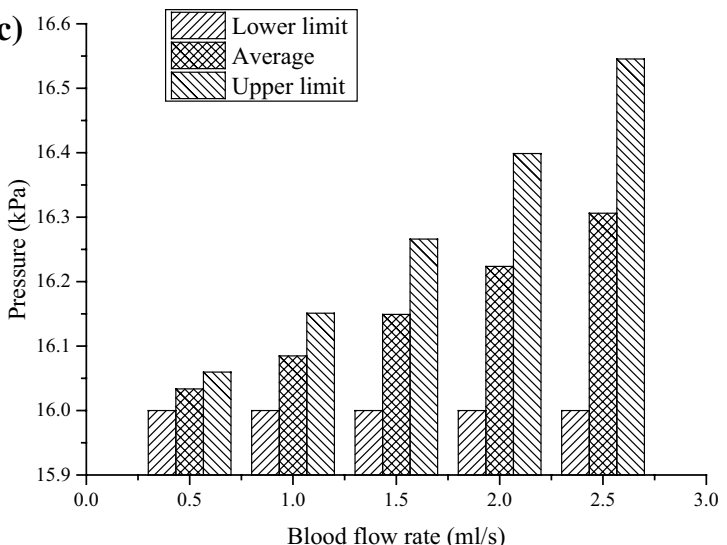

Fig. 5 VWS under different blood flow rates. a Distribution of WSS, b limit values for WSS, $\mathbf{c}$ limit values for wall pressure

\subsection{Combined influence}

To approximate a normal physiological state, the pulsatile blood flow rate and aortic pressure from Fig. 2 were applied as the inlet and outlet boundaries, respectively. The Carreau-Yasuda model (Eq. 4) was used to accommodate the shear thinning behavior of blood and vascular elasticity was fixed at $1 \mathrm{MPa}$. To examine the combined influence of the different factors, the distribution of WSS was assessed under conditions of the normal artery, rigidwall and Newtonian blood flow (Fig. 8). Two extreme moments ( $t=0.04$ and $t=0.2$ ) were selected for different flow state.

When blood flow rate decreased, the lower limit of WSS went down by $0.46 \mathrm{~Pa}$. Given the fact that blood pressure decreased and blood viscosity increased during the process, blood pressure and viscosity would both lead to an increase in WSS. In Fig. 8d, the biggest difference of lower limit between the normal model and rigid-wall model was around $0.15 \mathrm{~Pa}$ when flow rate reached its highest. The difference between normal model and Newtonian-blood model was about $0.1 \mathrm{~Pa}$ at both moments, and since the viscosity of non-Newtonian blood is always higher than
Newtonian blood, the effect on WSS caused by blood shear-thinning properties should be smaller than $0.1 \mathrm{~Pa}$. Therefore, the total reduction of WSS caused by a lower flow rate should be around $0.71 \mathrm{~Pa}$, indicating blood flow rate played a decisive role in the changes of WSS under a normal physiological state.

\section{Discussion and conclusion}

The human body is a unified whole and a small change can have a huge impact over time. After decades of accumulation of lipids in the intima, atherosclerosis can clog the vessel and endanger life. The formation of atherosclerosis is very complicated and associated with many factors, such as age, gender, diet and certain diseases $[28,29]$. There are studies showing that people with hypertension and hyperlipidemia are more likely to develop atherosclerosis [30, 31]; however, the mechanism by which these physiological conditions are linked to atherosclerosis is still unclear. In this work, by investigating the influence of physiological parameters on VWS, we were able to establish a connection between these parameters and hemodynamic 
(a)

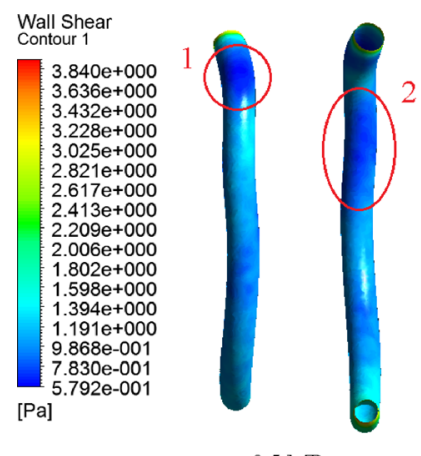

$0.5 \mathrm{MPa}$

(b)

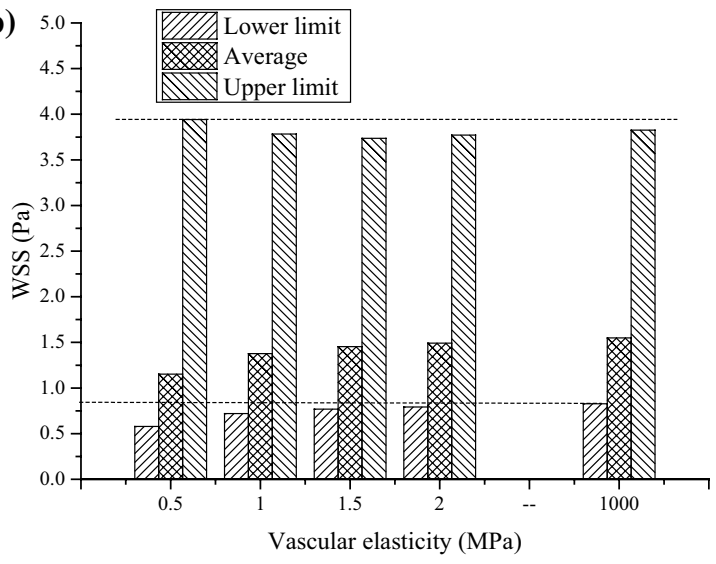

(1)

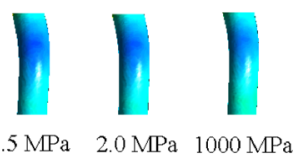

(2)

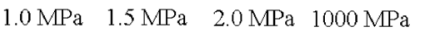

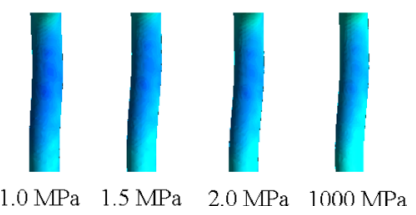

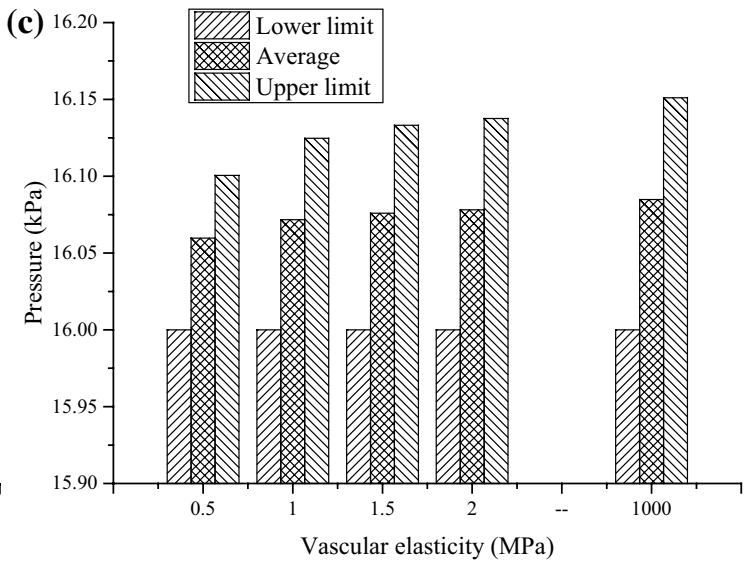

Fig. 6 VWS under different vascular elasticity. a Distribution of WSS, $\mathbf{b}$ limit values for WSS, c limit values for wall pressure

factors. The link between abnormal physiological conditions and atherosclerosis risk was explained in terms of hemodynamics.

Our data show that even though blood pressure can directly affect the pressure on the vessel wall, alone it had no influence on WSS. Nonetheless, when combined with a deformable vessel wall, blood pressure could indirectly influence WSS. However, the effect was very limited, only about $0.036 \mathrm{~Pa}$ for every $\mathrm{kPa}$ increase. Blood flow rate changes constantly in vivo, and it had a great impact on both WSS and wall pressure. When flow rate went down, WSS decreased in a near linear fashion. Additionally, in a normal physiological state, blood flow rate played a decisive role in the variation of WSS, indicating that it is a principal risk factor for atherosclerosis. Although vascular elasticity deterioration has long been recognized as an important cause of atherosclerosis in the elderly, the data showed only a minor effect of vascular elasticity on WSS. When the vessel wall became stiff, low WSS increased slightly and then leveled off, which could explain the limited effect of blood pressure on WSS. Similar to the blood flow rate, blood viscosity also had a great influence on WSS and wall pressure. A higher viscosity was associated with a notable increase in WSS; however, because of the shear-thinning behavior of blood, variations in viscosity in vivo only had a minor influence on WSS.

So through the results, it can be seen that high blood pressure, low blood flow rate, good elasticity of vessel wall and low blood viscosity could decrease WSS, which according to the low WSS theory [32-35] will promote the formation of atherosclerosis. The first two parameters are in accordance with what is observed in patients, while the latter two are not. That is because these parameters do not work in isolation in the body. Although a loss of elasticity and increased blood viscosity can cause slight increases in WSS, they also notably increase blood pressure (Figs. 6c, 7c) which would increase the load on the heart, causing the blood flow rate to decrease. And since the blood flow rate is the dominant factor in WSS, these changes in elasticity and viscosity will decrease WSS more than they will increase it, and along with it, the risk of atherosclerosis. Moreover, besides the effect on WSS, high blood pressure can also affect the permeability of lipids by dilating blood vessels, increasing the space between endothelial cells. One fact is that atherosclerotic lesions do not develop in veins, but they do indeed when the veins are used as arterial bypass grafts where they are subjected to high 
(a)

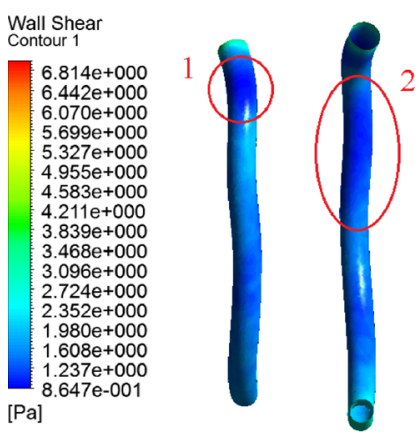

0.0035 Pas
(1)

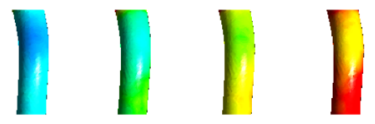

0.007 Pas 0.014 Pas 0.021 Pas 0.028 Pas

(2)

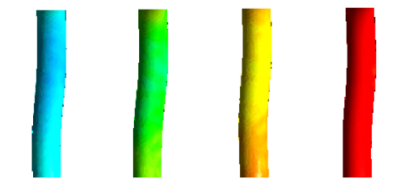

0.007 Pas 0.014 Pas 0.021 Pas 0.028 Pas (b)

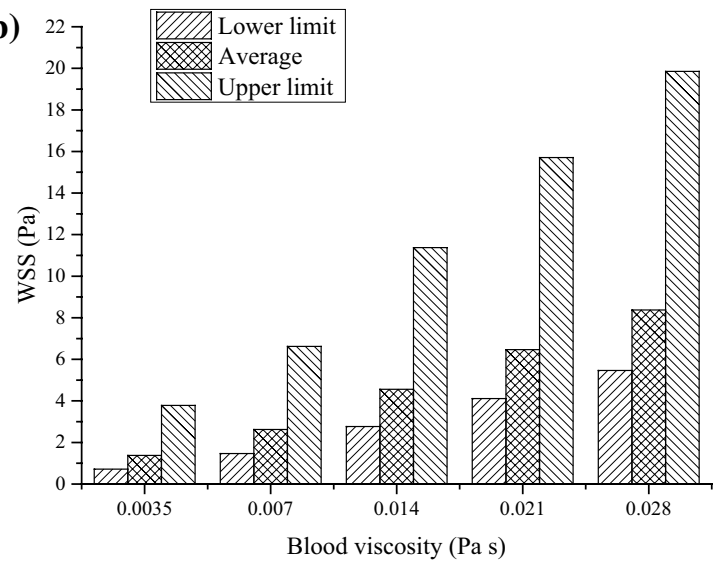

(c)

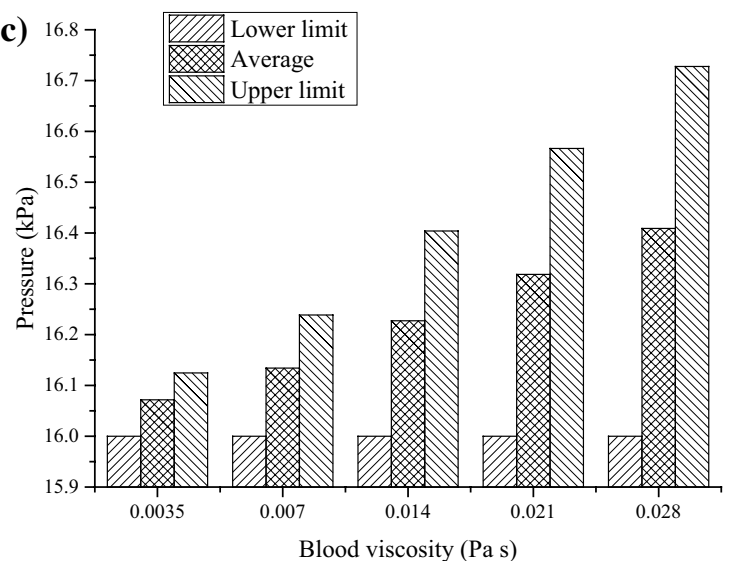

Fig. 7 VWS under different blood viscosity. a Distribution of WSS, $\mathbf{b}$ limit values for WSS, $\mathbf{c}$ limit values for wall pressure

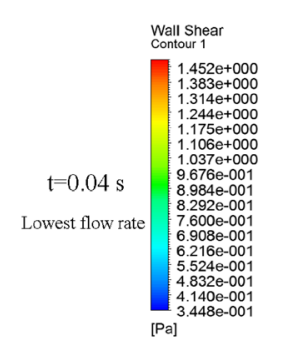

(a)

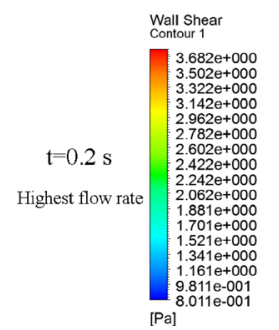

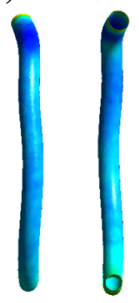

(b)

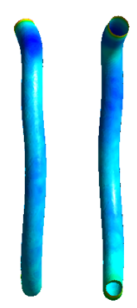

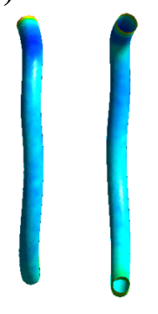

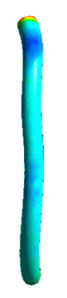

(c)

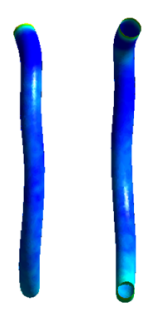

(d)

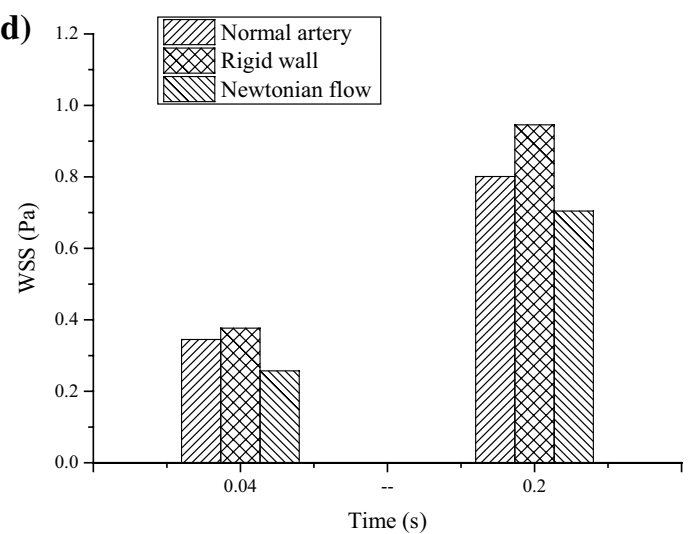

Fig. 8 Distribution of WSS under pulsatile conditions. a Normal artery, $\mathbf{b}$ rigid wall, $\mathbf{c}$ Newtonian blood, $\mathbf{d}$ limit values for WSS

pressure [36]. Thus, low blood flow rate and high blood pressure are both critical risk factors for atherosclerosis.

There are several limitations to our study. Although WSS is considered a major factor of atherosclerosis, there are still some other views and theories [1, 37]. Our conclusion is based on the low-WSS theory, and it matches well with clinical research. Even if there is new evidence in the future to support other theories, the influences of physiological 
parameters on wall stress, as in this study, are still valid. Moreover, we have made some simplifications to the calculation, such as ignoring side-branches and the assumption of vascular isotropic elasticity, which may have caused a certain deviation from the actual. However, the influence is limited; consistent with other research. This shows that our results are credible.

\section{Compliance with ethical standards}

Conflict of interest The authors declare that they have no conflict of interest.

\section{References}

1. Ross R (1999) Atherosclerosis-an inflammatory disease. N Engl J Med 340(2):115

2. Chaichana T, Sun Z, Jewkes J (2011) Computation of hemodynamics in the left coronary artery with variable angulations. J Biomech 44(10):1869-1878

3. Napoli C, D'Armiento FP, Mancini FP et al (1997) Fatty streak formation occurs in human fetal aortas and is greatly enhanced by maternal hypercholesterolemia. Intimal accumulation of low density lipoprotein and its oxidation precede monocyte recruitment into early atherosclerotic lesions. J Clin Investig 100(11):2680-2690

4. Crouse JR, Goldbourt U, Evans G et al (1996) Risk factors and segment-specific carotid arterial enlargement in the Atherosclerosis Risk in Communities (ARIC) cohort. Stroke 27(1):69-75

5. Dharmashankar K, Widlansky ME (2010) Vascular endothelial function and hypertension: insights and directions. Curr Hypertens Rep 12(6):448

6. Newman WP 3rd, Freedman DS, Voors AW et al (1986) Relation of serum lipoprotein levels and systolic blood pressure to early atherosclerosis. The Bogalusa Heart Study. N Engl J Med 314:138-144

7. Lakka TA, Salonen R, Kaplan GA et al (1999) Blood pressure and the progression of carotid atherosclerosis in middle aged men. Hypertension 34:51-56

8. Kamarck TW, Everson SA, Kaplan GA et al (1997) Exaggerated blood pressure responses during mental stress are associated with enhanced carotid atherosclerosis in middle-aged finish men: findings from the Kuopio Ischemic Heart Disease Study. Circulation 96:3842-3848

9. Sander D, Kukla C, Klingelhöfer J et al (2000) Relationship between circadian blood pressure patterns and progression of early carotid atherosclerosis: a 3-year follow-up study. Circulation 102(13):1536-1541

10. Carallo C, Pujia A, Irace C et al (1998) Whole blood viscosity and haematocrit are associated with internal carotid atherosclerosis in men. Coron Artery Dis 9(2-3):113-117

11. Vosseler M, Beutel A, Schäfer S et al (2012) Parameters of blood viscosity do not correlate with the extent of coronary and carotid atherosclerosis and with endothelial function in patients undergoing coronary angiography. Clin Hemorheol Microcirc 52(2-4):245

12. Lowe GD, Lee AJ, Rumley A et al (2015) Blood viscosity and risk of cardiovascular events: the Edinburgh Artery Study. Br J Haematol 96(1):168-173
13. Davies PF, Tripathi SC (1993) Mechanical stress mechanisms and the cell. An endothelial paradigm. Circ Res 72(2):239-245

14. Weinbaum S, Chien S (1993) Lipid transport aspects of atherogenesis. J Biomech Eng 115(4B):602

15. Fung YC, Liu SQ (1993) Elementary mechanics of the endothelium of blood vessels. J Biomech Eng 115(1):1

16. Schmidschoenbein GW, Fung YC, Zweifach BW (1975) Vascular endothelium-leukocyte interaction: sticking shear force in venules. Circ Res 36(1):173-184

17. Salmon S, Thiriet M, Gerbeau JF (2003) Medical image-based computational model of pulsatile flow, in saccular aneurisms. ESAIM Math Model Numer Anal 37(4):663-679

18. Steinman DA (2002) Image-based computational fluid dynamics modeling in realistic arterial geometries. Ann Biomed Eng 30(4):483-497

19. Zhao $S Z, X u X Y$, Hughes $A D$ et al (2000) Blood flow and vessel mechanics in a physiologically realistic model of a human carotid arterial bifurcation. J Biomech 33(8):975-984

20. Holzapfel GA, Sommer G, Gasser C, Regitnig P (2005) Determination of layer-specific mechanical properties of human coronary arteries with nonatherosclerotic intimal thickening and related constitutive modeling. Am J Physiol Heart Circ Physiol 289(5):H2048-H2058

21. Toloui M, Firoozabadi B, Saidi MS (2012) A numerical study of the effects of blood rheology and vessel deformability on the hemodynamics of carotid bifurcation. Scientia Iranica 19(1):119-126

22. Leuprecht A, Perktold K (2001) Computer simulation of nonnewtonian effects on blood flow in large arteries. Comput Methods Biomech Biomed Eng 4(2):149-163

23. Abraham F, Heinkenschloss MB (2005) Shape optimization in unsteady blood flow: a numerical study of non-Newtonian effects. Comput Methods Biomech Biomed Eng 8(2):127-137

24. Soulis JV, Fytanidis DK, Lampri OP et al (2016) Low density lipoprotein and non-Newtonian oscillating flow biomechanical parameters for normal human aorta. Cardiol Res 7(2):66-79

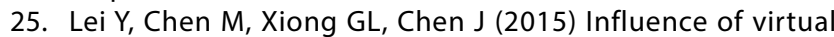
intervention and blood rheology on mass transfer through thoracic aortic aneurysm. J Biomech 48(12):3312-3322

26. Liu GY (2014) Computational simulation of hemodynamic characteristics in human right coronary arteries. Southern Medical University, Guangdong

27. Wada S, Karino T (2002) Theoretical prediction of low-density lipoproteins concentration at the luminal surface of an artery with a multiple bend. Ann Biomed Eng 30(6):778-791

28. Katakami N (2018) Mechanism of development of atherosclerosis and cardiovascular disease in diabetes mellitus. J Atheroscler Thromb 25(1):27-39

29. Duivenvoorden R (2019) Atherosclerosis and diabetic nephropathy. In: Roelofs JJ, Vogt L (eds) Diabetic nephropathy. Pathophysiology and clinical aspects. Springer, Cham, pp 357-372

30. Genkel VV, Salashenko AO, Shamaeva TN et al (2018) Association between carotid wall shear rate and arterial stiffness in patients with hypertension and atherosclerosis of peripheral arteries. Int J Vasc Med 2018:1-8

31. Liu B, Chen Z, Dong X et al (2018) Association of prehypertension and hyperhomocysteinemia with subclinical atherosclerosis in asymptomatic Chinese: a cross-sectional study. BMJ Open 8(3):e019829

32. Sumpio BE, Banes AJ, Levin LG et al (1987) Mechanical stress stimulates aortic endothelial cells to proliferate. J Vasc Surg 6(3):252-256

33. Thubrikar MJ, Moorthy RR, Deck JD et al (1994) Smooth muscle cell proliferation: is it due to endothelial injury or aortic stretch. Atherosclerosis 109(1-2):100 
34. Clowes AW, Clowes MM, Reidy MA (1987) Role of acute distension in the induction of smooth muscle proliferation after endothelial denudation [Abstract]. Fed Proc 46:720

35. Sumpio BE, Banes AJ, Buckley M, Johnson G (1988) Alteration in aortic endothelial cell morphology and cytoskeletal protein synthesis during cyclic tensional deformation. J Vasc Surg 7:130-138

36. Thubrikar MJ, Robicsek F (1995) Pressure-induced arterial wall stress and atherosclerosis. Ann Thorac Surg 59(6):1594-1603
37. Fry DL (1968) Acute vascular endothelial changes associated with increased blood velocity gradients. Circ Res 22(2):165-197

Publisher's Note Springer Nature remains neutral with regard to jurisdictional claims in published maps and institutional affiliations. 